\title{
DNA-Mediated Fluctuations in Ionic Current through Silicon Oxide Nanopore Channels
}

\author{
H. Chang, ${ }^{\dagger}$ F. Kosari,,$\S$ G. Andreadakis,,$\$$ M. A. Alam, ${ }^{\dagger}$ G. Vasmatzis,,${ }^{*} \S$ and \\ R. Bashir ${ }^{\star},+, \ddagger$
}

\begin{abstract}
School of Electrical and Computer Engineering, Purdue University, West Lafayette, Indiana, Department of Biomedical Engineering, Purdue University, West Lafayette, Indiana, and Department of Laboratory Medicine and Pathology, Division of Experimental Pathology, Mayo Clinic, Rochester, Minnesota
\end{abstract}

Received May 16, 2004; Revised Manuscript Received June 11, 2004

\begin{abstract}
Single molecule sensors in which nanoscale pores within biological or artificial membranes act as mechanical gating elements are very promising devices for the rapid characterization and sequencing of nucleic acid molecules. The two terminal electrical measurements of translocation of polymers through single ion channels and that of ssDNA molecules through protein channels have been demonstrated, and have sparked tremendous interest in such single molecule sensors. The prevailing view regarding the nanopore sensors is that there exists no electrical interaction between the nanopore and the translocating molecule, and that all nanopore sensors reported to-date, whether biological or artificial, operate as a coulter-counter, i.e., the ionic current measured across the pore decreases (is mechanically blocked) when the DNA molecule transverses through the pore. We have fabricated nanopore "channel" sensors with a silicon oxide inner surface, and our results challenge the prevailing view of exclusive mechanical interaction during the translocation of dsDNA molecules through these channels. We demonstrate that the ionic current can actually increase due to electrical gating of surface current in the channel due to the charge on the DNA itself.
\end{abstract}

As a first step toward the ultimate goal of single-molecule DNA sequencing using nanopore sensors, one must first identify the key mechanical and electrical variables that control the translocation of the molecules through the nanopores. First, a nanopore used for characterization and sequencing of a single molecule must have a diameter of less than the persistence length $(\sim 50 \mathrm{~nm}$ for dsDNA) to avoid any signal averaging from thermally induced conformational changes. Second, the pores must be chemically stable and mechanically robust under a wide variety of conditions of use. Third, and finally, the mechanical and electrical interaction between the nanopores and the single molecules must be well characterized. Toward this end, the electrical detection of translocation of polymers through single ion channels has been demonstrated. ${ }^{1-2}$ Subsequently, the pioneering studies of the use of $\alpha$-hemolysin protein pore within a lipid bilayer for the translocation of single strands of DNA molecules using a voltage bias across the membranes sparked tremendous interest in such single molecule sensors. ${ }^{3-8}$

\footnotetext{
* Corresponding authors. E-mail: bashir@purdue.edu or vasm@ mayo.edu

School of Electrical and Computer Engineering, Purdue University.

$\doteqdot$ Department of Biomedical Engineering, Purdue University.

$\S$ Mayo Clinic.
}

The normal ionic current through the protein pore in a lipid bilayer would detectably reduce as a polyanionic chain of ssDNA molecules traversed through the pore, even allowing the distinction between polycytosine and polyadenine molecules, thus demonstrating the potential of single base discrimination in these sensors. ${ }^{4,5}$

Despite these advantages, robust integration of these biological sensors within practical devices is quite problematic, and a mechanical pore ${ }^{9,10}$ provides numerous advantages over biological pores. Besides the obvious advantages of being able to drastically change ambient conditions such as $\mathrm{pH}$, electric field, and temperature without distorting the system, such a system can also allow the integration of different types of electronic or optical sensors ${ }^{11}$ or functionalization of the inner surface of the pore, as demonstrated for protein channels for the detection of single chemical molecules and analytes. ${ }^{12-14}$ In addition, the use of top-down fabricated nanopore sensors promises robust integrated nanofluidic devices for applications in single cell expression analysis, highly sensitive chemical and biological detection, nanobioelectronics, and nanosystems biology.

Recent reports of the use of ion milling ${ }^{7}$ and transmission electron beam imaging ${ }^{15,16}$ with real time feedback to form controlled pore size have paved the way to begin the 


\section{Top View TEM image}

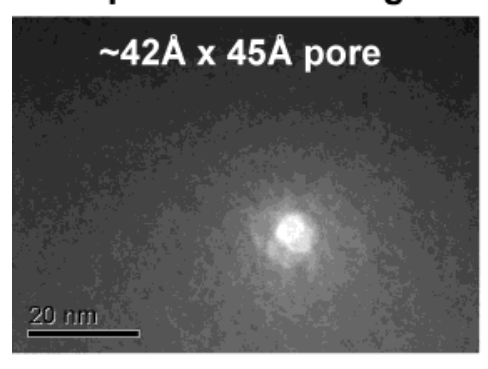

(a)

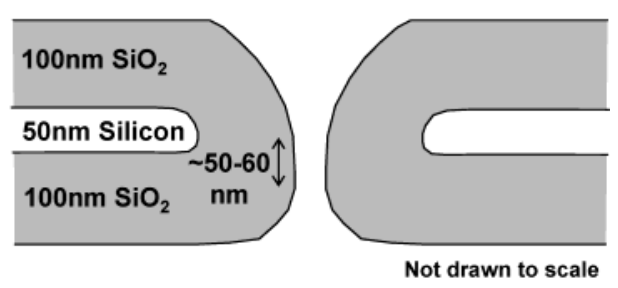

(b)

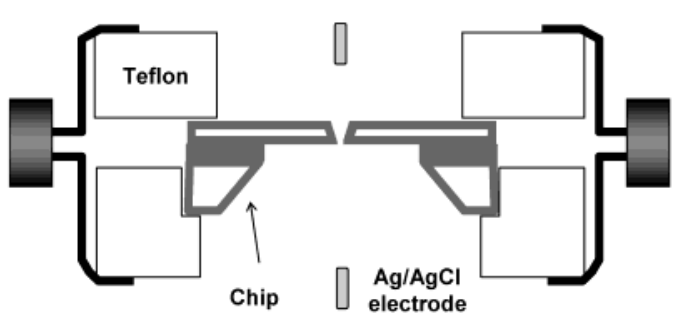

(c)

Figure 1. (a) Transmission electron microscope (TEM) image of the smallest diameter of the nanopore channel in the oxidized silicon membrane, fabricated as described in the text. Due to the dimensions shown, the expected channel length is around 50-60 $\mathrm{nm}$. (b) Drawn cross-section of the oxidized silicon membrane. (c) Schematic of the apparatus that shows the entire chip within Teflon reservoirs ensuring no fluid leaks across the chambers. The current was measured using Ag/AgCl electrodes. The diaphragm was $80 \mu \mathrm{m} \times 80 \mu \mathrm{m}$.

exploration of single DNA molecule translocations and conformational studies using artificial pores. The electronic signature of dsDNA moving through these nanopores can be quite complex and the conformational changes in the molecule cannot be ignored, as shown recently by Li et al. ${ }^{9}$ One possibility to suppress signatures due to conformational changes is to increase the entropic energy barrier for molecules to enter the nanopore by the use of "channels" with nanoscale diameters. ${ }^{17}$ We fabricated 50-60 nm long, 4-5 $\mathrm{nm}$ diameter nanopore channels in micromachined silicon membranes. ${ }^{16}$ The nanopore channels were fabricated with a process similar to one described earlier ${ }^{15}$ and commenced with double polished SOI wafers. ${ }^{16}$ The buried oxide and SOI layers were 400 and $190 \mathrm{~nm}$ thick, respectively. A $100 \mathrm{~nm}$ thermal oxide was grown on the SOI wafer. An LPCVD nitride layer was deposited and etch windows ( $\sim 670 \mu \mathrm{m} \times 670 \mu \mathrm{m}$ area) were formed on the backside. The nitride and oxide were etched, and the handle layer of the wafer was etched from the backside in TMAH at $90{ }^{\circ} \mathrm{C}$ to stop on the buried oxide to form a diaphragm of around $80 \mu \mathrm{m}$ by $80 \mu \mathrm{m}$ area. The nitride was stripped off in boiling phosphoric acid, and electron beam lithography was performed on the front to define $100 \mathrm{~nm} \times 100 \mathrm{~nm}$ openings with the diaphragm. Next, a short TMAH etch at $60^{\circ} \mathrm{C}$ was performed to etch the SOI, in an inverted pyramid shape, down to the buried oxide. When the buried oxide is removed, a pore of less than $100 \mathrm{~nm}$ in diameter is formed. A $1000 \AA$ thick thermal oxide was then grown to shrink the pore to less than $50 \mathrm{~nm}$. Finally, the pore was examined and processed in a transmission electron micrograph and shrunk to the desired nanometer dimension utilizing direct observation. Figure 1a shows the final TEM of the small dimension of a nanopore channel, while Figure 1b shows the expected cross-section of the channel with the relevant dimensions at the end of fabrication.

Each nanopore channel, fabricated according to the process described above, was formed within a die that was $3 \mathrm{~mm} \times$ $3 \mathrm{~mm}$ in size and about $0.5 \mathrm{~mm}$ thick. The die with the nanopore was sandwiched between two silicone rings and placed in a finely milled pocket within Teflon blocks with the chambers and subsequently clamped, as shown in Figure 1c. Electrophysiology measurements were performed using an Axon 200B amplifier (Axon Instruments) in the resistive feedback mode, and a CV201 head stage. To reduce the environmental noise, the nanopore and the head stage were placed inside a grounded Faraday cage. The amplifier signal was passed through a $300 \mathrm{~Hz}$ low-pass filter (CyberAmp 320) and fed to a Digidata 1322A (Axon Instruments) board on computer with PClamp 9.0 (Axon Instrument) software for data acquisition.

A 200 bp fragment from the human CRISP-3 gene (accession number NM_006061) was PCR amplified (forward primer, TCCTGCTGGTAATTGGGCTAA, reverse primer, TGTCCCTGACCAACTGATGTTT) using cDNA from a prostate cancer specimen and purified by the PCR purification kit (Qiagen, Valencia, CA).The measurements were carried out in $0.1 \mathrm{M} \mathrm{KCl}, 2 \mathrm{mM}$ Tris ( $\mathrm{pH} 8.5$ ) buffer. To prevent isolation of the nanopore by air bubbles, 2-propanol was applied to the exposed surface of the nanopore prior to applying the buffer. After some time, purified DNA at a final concentration of $0.3 \mu \mathrm{g} / \mathrm{mL}$ was applied to one chamber (side 1) and the current measurements from that chamber to the other chamber (side 2) were made. $\mathrm{Ag} / \mathrm{AgCl}$ electrodes were inserted in the fluid reservoirs and used to make the measurements. 


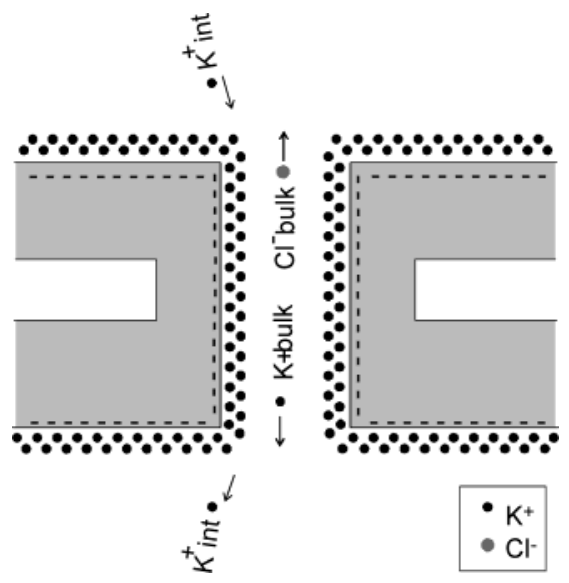

Figure 2. Schematic of the expected nanopore channel depicting potassium ions accumulated close to the negatively charged oxide surface, before the DNA is passed through.

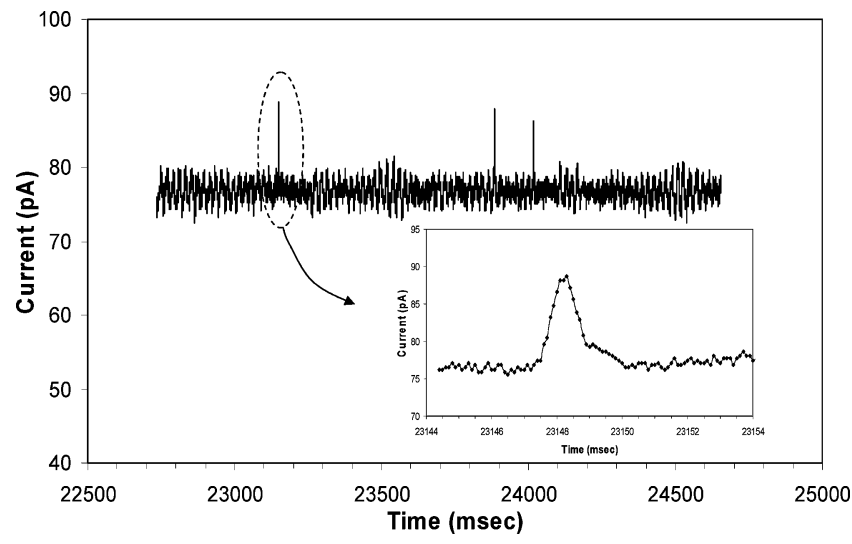

Figure 3. Translocation of the $200 \mathrm{bp}$ dsDNA molecule. Typical pulses measured for the translocation of the short DNA through the nanopore channel are shown. The inset shows the one pulse in more detail.

The electrical response of such nanopores in the absence of DNA translocation depends on geometry and electrical charge distribution within the pore itself. It is well known that the oxide surface of the nanopore channel would become strongly negatively charged around $\mathrm{pH} 7$ due to deprotonation of the silanol $(\mathrm{SiOH})$ groups. $^{18}$ The negative surface charge density due to these $\mathrm{SiO}^{-}$groups can be up to (4-5) $\times 10^{14}$ charges $/ \mathrm{cm}^{2} .{ }^{19}$ This translates to a maximum of

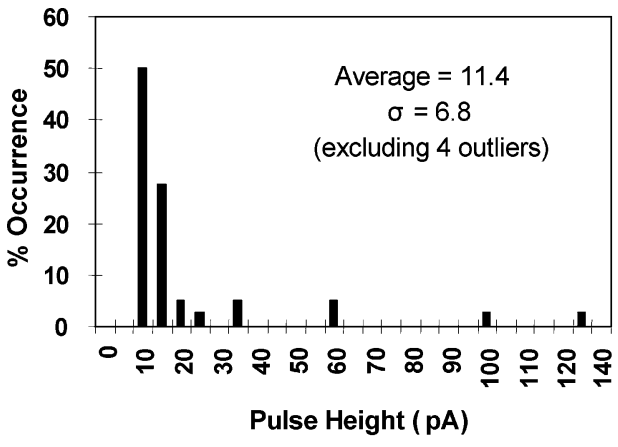

(a) $\sim 3500$ negative charges $\left(5.6 \times 10^{-16} \mathrm{C}\right)$ in the inner surface of the channel. ${ }^{20}$ These charges create a screening layer of mobile $\mathrm{K}^{+}$ions concentrated close to the surface which can take part in current flow under an electric field applied parallel to the length of the pore (same effect as electroosmosis). During steady-state conditions without DNA, we expect three current components, as shown in Figure 2. There will be bulk $\mathrm{K}^{+}$and $\mathrm{Cl}^{-}$currents, $I_{\text {bulk }}\left(\mathrm{K}^{+}\right)$and $I_{\text {bulk }}\left(\mathrm{Cl}^{-}\right)$, and an interfacial layer $\mathrm{K}^{+}$current, $I_{\mathrm{int}}\left(\mathrm{K}^{+}\right)$. It should be pointed out that even though the distinction between bulk and interface current is not clear due to the nanoscale diameter of the channel, we chose to make these distinctions for purposes of discussion and explanation of the results.

We measured steady-state ionic currents in the range of 55-70 pA through the nanopore channel with $200 \mathrm{mV}$ in a $0.1 \mathrm{M} \mathrm{KCl}$ solution. ${ }^{22} \mathrm{We}$ applied $200 \mathrm{bp}$ dsDNA to side 1 and as the dsDNA passed through the pore, it modified the bulk, and the interface currents resulting in typical current pulses are shown in Figure 3. However, unexpectedly and in contrast to previous reports, we find the signal current to increase rather than decrease during the translocation of dsDNA through the nanopore. The average pulse height and pulse width for 40 pulses is about $11.4 \mathrm{pA}$ and $4 \mathrm{~ms}$, respectively, as shown in Figure $4 \mathrm{~b}$ and c. It can be pointed out that the DNA can affect the current in two ways: (i) by changing the cross-sectional area and (ii) by changing the carrier concentration. Hence, we attribute the surprising increase in current to the following mechanism (also shown in Figure 5a,b). As the dsDNA molecule enters the channel, the bulk currents $I_{\text {bulk }}\left(\mathrm{K}^{+}\right)$and $I_{\text {bulk }}\left(\mathrm{Cl}^{-}\right)$will decrease due to the physical blocking by the DNA molecule, as usually expected. With given channel radius of $2.2 \mathrm{~nm}$ and dsDNA radius of $1.1 \mathrm{~nm}$, we expect the blocking to reduce the current by $\sim 25 \%$ (from $75 \mathrm{pA}$ to $56 \mathrm{pA}$ or $\Delta I_{\text {bulk }}\left(\mathrm{K}^{+}\right)+\Delta I_{\text {bulk }}\left(\mathrm{Cl}^{-}\right)$ $\sim-19 \mathrm{pA}$ ). However, since the DNA backbone is negatively charged, its translocation will also induce additional $\mathrm{K}^{+}$ atoms within the channel, and therefore the interface current, $I_{\text {int }}\left(\mathrm{K}^{+}\right)$, will actually increase. It should be noted that the concentration of positively charged potassium ions inside or in close proximity to the channel is much higher than the bulk solution. Assuming that all interface charge is neutralized, the concentration of potassium ions is equivalent to 76 $\mathrm{M}$ ! This is clearly not realistic, and any of the following

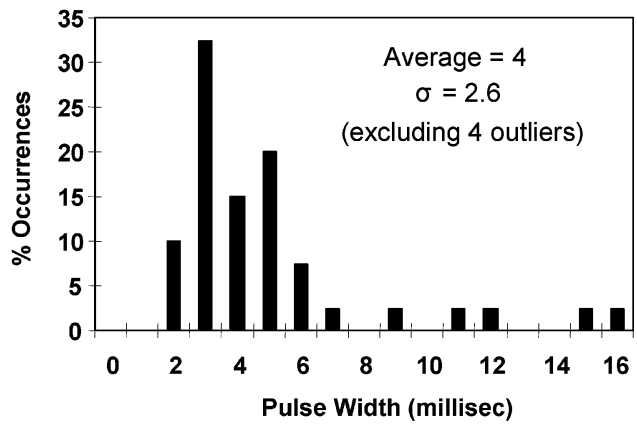

(b)

Figure 4. The histograms of raw data for (a) pulse height, and (b) width for 40 pulses; average and standard deviations, excluding four outliers, are also shown. 


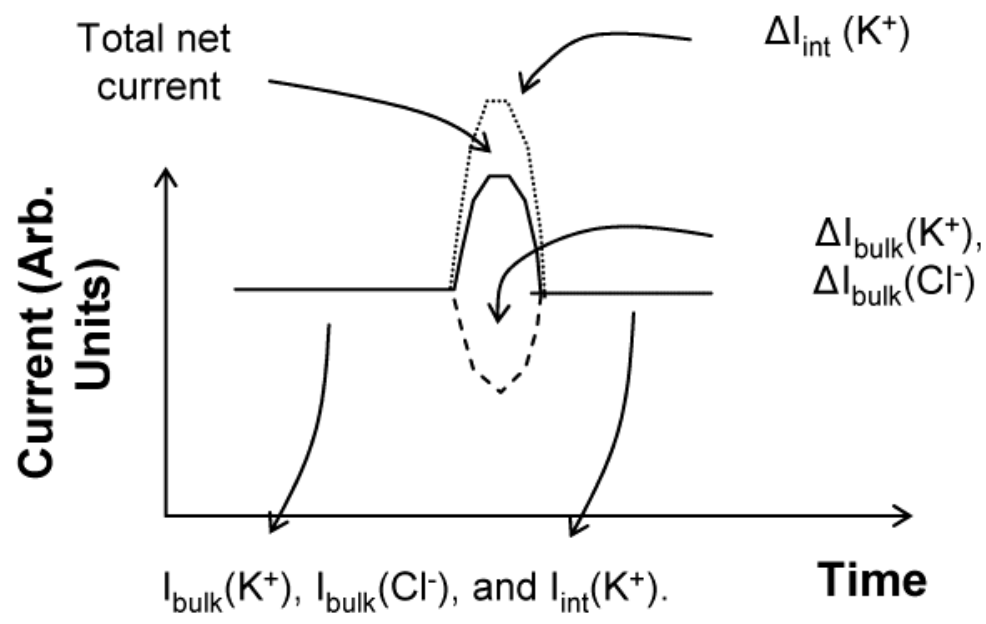

(a)

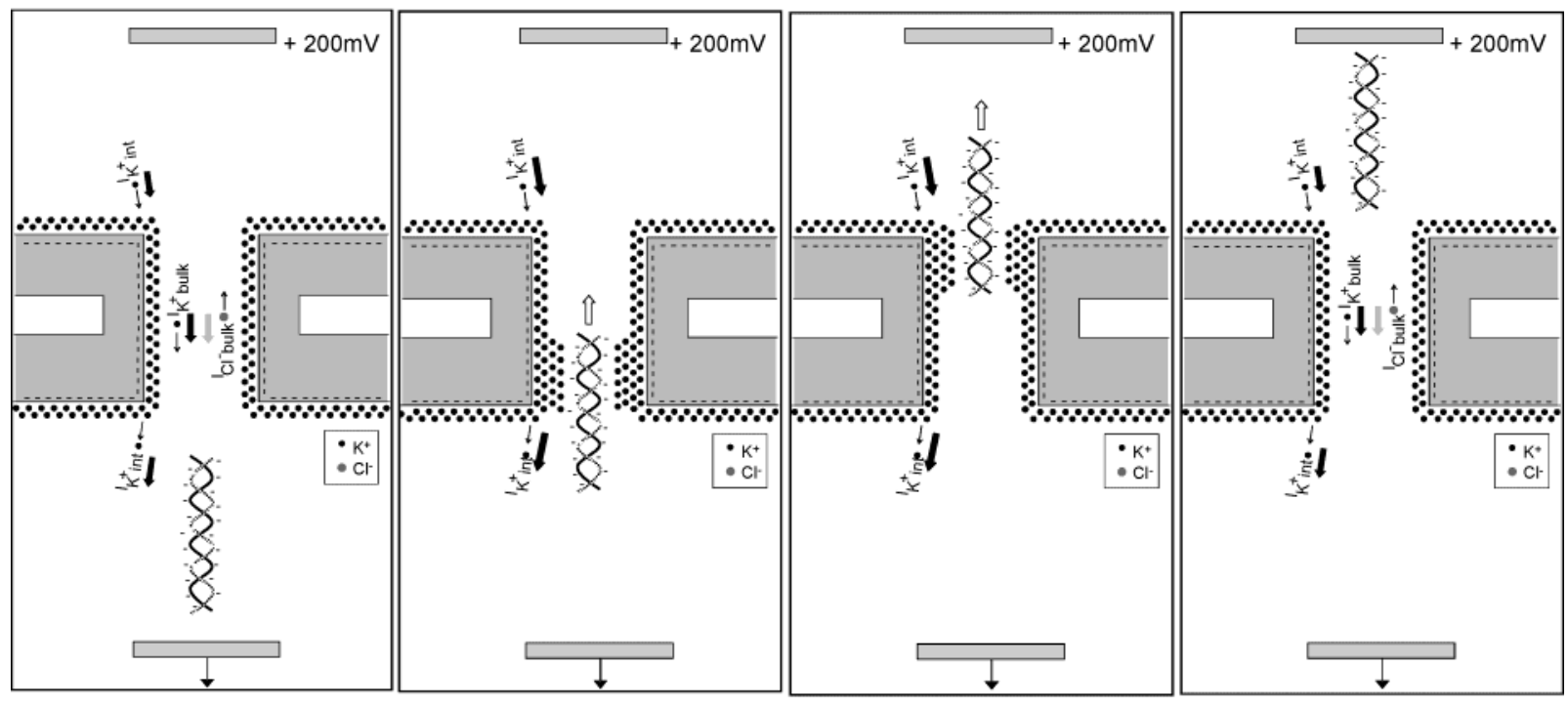

Figure 5. Schematic of the DNA molecule threading through the pore. (a) Qualitative explanation of the pulse shape. With the DNA, the baseline current consists of three components as shown and explained in the text. (b) Once the DNA enters the pore, the bulk ionic current decreases due to mechanical blocking, while the interfacial current through the mobile layer increases. Since the molecule is about the same length as the channel, as soon as the current reaches a maximum, the molecule will begin to leave the pore and the current should decrease, as experimentally measured. The blocking current, in this case, will overlap with the increase in the interfacial current. As the molecule completely exits the pore, the blocking current recovers and the interfacial current decreases. The interface potassium current is expected to increase when the DNA is threading through the nanopore channel and reaches a maximum when the pore is fully occupied by the DNA.

scenarios are possible: (i) not all the negative surface charges are neutralized locally, and the counterions are not inside the channel but rather are present at the edges of the channel, or (ii) the negative fixed charge density is less than the maximum value assumed. The point to note is that as the DNA enters the pore, the higher potassium ion concentration will tend to reduce the net charge on the DNA, more than in the bulk solution region. The excess positive charge, thus, contributes to an increase in the interfacial current (the channel can provide a novel device for temporally varying the ionization of the DNA molecule). The role of the DNA molecule in this case is analogous to the gate of a field effect transistor that causes the interfacial layer channel charge to increase, resulting in an increased current flow due to the voltage applied across the pore. Since the DNA molecule is around the same length as the channel, the current is expected to increase and then decrease as the DNA leaves the pore, as shown in the measured results. We can use simple expressions to estimate the peak current induced due to the $\mathrm{K}^{+}$ions in the accumulated channel as

$$
\Delta \mathrm{I}_{\mathrm{int}}\left(\mathrm{K}^{+}\right)=A n q \mu_{\mathrm{K}^{+}}(b \cdot V / L)
$$

where, $A$ is the cross-sectional area of the channel minus the cross-sectional area of the DNA, $n$ is the induced charge density, $q$ is the electron charge, $\mu_{K^{+}}$is the mobility of the potassium ion, $V / L$ is the electric field across the channel, and $b$ is a parameter between 0.67 and 1 . This parameter accounts for the fact that the applied voltage at the electrodes does not all appear across the nanopore channel, rather a certain fraction of the applied voltage does. Initial continuum simulations of our structures show that roughly two-thirds 
of the voltage applied at the electrodes appears across the nanopore channel and hence we will use 0.67 for $b$ in the calculations. The induced charge density $n$ can be calculated by dividing the number of additional charges by the volume of the nanopore channel minus the volume taken by the dsDNA molecule. The induced charge is maximum at about $130 \mathrm{e}$ (assuming e/3 for each phosphate ion, ${ }^{10}$ noting that this can change inside the nanopore channel due to the presence of the high $\mathrm{K}^{+}$ion concentration, as discussed earlier) when the dsDNA is fully inside the channel, thus $n \sim 2.28 \times 10^{20}$ $\# / \mathrm{cm}^{3}$ (assuming $50 \mathrm{~nm}$ long channel with radius of $2.2 \mathrm{~nm}$ ). We can use $V / L=200 \mathrm{mV} / 50 \mathrm{~nm}=4 \times 10^{4} \mathrm{~V} / \mathrm{cm}$ and $A$ $=\pi\left(R^{2}-R_{\mathrm{DNA}}^{2}\right)$, where $R$ is the radius of the nanopore channel of $2.2 \mathrm{~nm}$, and $R_{\mathrm{DNA}}$ is the radius of the dsDNA assumed to be $1.1 \mathrm{~nm}$. The value of mobility of potassium ions in aqueous $\mathrm{KCl}$ solution was used as $7.57 \times 10^{-4} \mathrm{~cm}^{2} / \mathrm{V}$ $\mathrm{s},{ }^{23}$ resulting in a calculated current value of $\Delta I_{\text {int }}\left(\mathrm{K}^{+}\right) \sim 84$ $\mathrm{pA}$. Therefore, the net current is $\sim 84 \mathrm{pA}-19 \mathrm{pA} \sim 65$ pA. Given the approximate nature of the calculation and the fact that we used low field mobility values, which do not reflect the reduction in mobility at high fields (could be reduced by $3-4 \times$ ), the calculated current ${ }^{24}$ is in the same order of the measured values as shown in Figure $2 \mathrm{a}$. Therefore, our results indicate that, in addition to the mechanical gating, which has thus far been assumed to be the only source of current fluctuation in nanopore sensors, the electrical gating of dsDNA could play a major role in determining the electrical pulse shape in the channel current, if a negatively charged oxide surface is used as the inside surface of the nanopore channel.

It should also be noted that the velocity of the $200 \mathrm{bp}$ DNA in our pores $\left(\sim 50 \mathrm{~nm} / 3 \mathrm{~ms}=1.6 \times 10^{-5} \mathrm{~m} / \mathrm{s}\right)$ is much slower than the velocity of DNA reported in $\mathrm{Si}_{3} \mathrm{~N}_{4}$ pores ${ }^{8,9}$ $\left(\sim 1.25 \times 10^{-2} \mathrm{~m} / \mathrm{s}\right.$ for $3000 \mathrm{bp}$ dsDNA at $\left.120 \mathrm{mV}\right)$ and about the same as the $\alpha$-hemolysin pore ${ }^{7}$ ( $\sim$ for $200 \mathrm{bp}$ ssDNA, at $2{ }^{\circ} \mathrm{C}$ ). Our pore is longer and is expected to have significant interaction with the DNA due to the electrostatic forces between the positive mobile charges and the negative DNA backbone charge, and due to the hydrodynamic drag of the electroosmotic current in the opposite direction as the movement of the DNA. We also note that the current carried by the DNA strand itself is negligible, as estimated by the time taken for translocation and the charge on the DNA.

To prove that some DNA molecules have indeed passed through the nanopore, after the experiment, we collected and concentrated the buffers from the two chambers and performed a PCR using the same forward and reverse primers that we used to amplify the DNA. We then ran the PCR products on an ethidium bromide gel and observed a strong band at the expected size from the buffer in side 1. More importantly, we also observed the same size band in the buffer from side 2, as shown in Figure 6, proving that some DNA molecules have in fact traveled through the nanopore from side 1 to side 2 .

The experimental results presented here demonstrate that the charge on the DNA can be detected in the nanopore channel due to an inherent charge amplification and transduction of the DNA charge into ionic current in the mobile

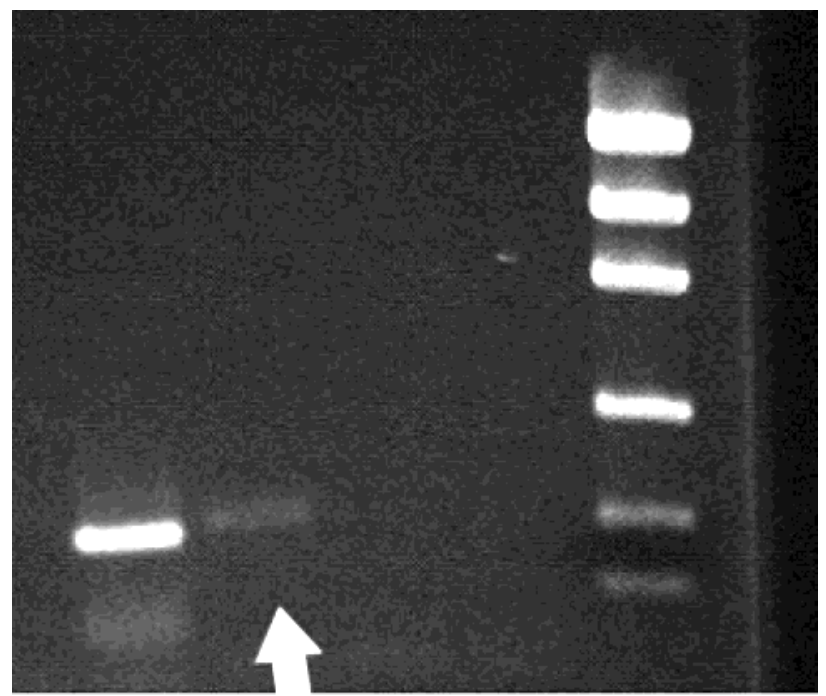

Figure 6. PCR products from the buffer in the two chambers. Lane 1 displays the expected band from the buffer in the chamber where DNA was applied (side 1). Lane 2 is the PCR product from the buffer in the other chamber (side 1). The right-most lane is the 100 bp DNA ladder.

surface charge, similar to a field effect transistor. Clearly, while more detailed experimental work and modeling needs to be performed, including use of longer molecules and improvements in device design such as controlling the interface oxide charge by biasing of the silicon layer, the demonstration of electrical gating effect would allow one to use these nanopore membranes as the third electrode capable of characterizing multiple threads of dsDNA simultaneously. The electrical signal from this device for the passage of short dsDNA molecules with mismatches could exhibit a difference when compared to the signal from matched strands, providing a label-free means to detect DNA hybridization. The silicon layer can possibly be used as the third electrode to measure changes in potential during the passage of DNA through the nanopore channel.

Acknowledgment. We acknowledge Dr. Ed Basgall at Penn State University for support in electron beam lithography, Prof. Alex King at the School of Materials Science and Engineering for training Hung Chang for use of TEM, the Birck Nanotechnology Center at Purdue University for wafer processing, and Dr. Dallas Morrisette, Dr. David Janes, Dr. Demir Akin, and Mr. Oguz Elibol for valuable discussions. We would also like to thank Dr. J. L. Rae and Dr. G. Farrugia at Mayo Clinic for the use of the measurement apparatus and for valuable discussions. The authors are indebted to the Rockefeller Brothers Pilot Grant and NIH (through R21 RR15118-01) for getting the work started and to the NASA Institute of Nanoelectronics and Computing (INAC) at Purdue University under award no. NCC 2-1363 for the funding to continue the work.

\section{References}

(1) Bezrukov, S.; Vodyanoy, I.; Parsegian, V. A. Nature 1994, 370, 279281. 
(2) Cornell, B. A.; Braach-Maksvytis, V. L. B.; King, L. G.; Osman, P. D. J.; Raguse, B.; Wiezorek, L.; Pace, R. J. Nature 1997, 387, 580583.

(3) Kasianowicz, J. J.; Brandin, E.; Branton, D.; Deamer, D. W. Proc. Natl. Acad. Sci. U.S.A. 1996, 93, 13770-13773.

(4) Akeson, M.; Branton, D.; Kasianowicz, J. J.; Brandin, E.; Deamer, D. W. Biophys. J. 1999, 77, 3227-3233.

(5) Meller, A.; Nivon, L.; Brandin, E.; Golovchenko, J.; Branton, D. Proc. Natl. Acad. Sci. U.S.A. 2000, 97, 1079-1084.

(6) Vercoutere, W.; Winters-Hilt, S.; Olsen, H.; Deamer, D.; Haussler, D.; Akeson, M. Nature Biotechnol. 2001, 19, 248-252.

(7) Meller, A.; Nivon, L.; Branton, D. Phys. Rev. Lett. 2001, 86, 34353438.

(8) Song, L.; Hobaugh, M. R.; Shustak, C.; Cheley, S.; Bayley, H.; Gouaux, J. E. Science 1996, 274, 1859-1865.

(9) Li, J.; Stein, D.; McMullan, C.; Branton, D.; Aziz, M. J.; Golovchenko, J. A. Nature 2001, 412, 166-169.

(10) Li, J.; Gershow, M.; Stein, D.; Brandin, E.; Golovchenko, J. Nature Materials 2003, 2, 611-615.

(11) Branton, D.; Golovchenko, J. Nature 1999, 398, 660.

(12) Gu, L. Q.; Braha, O.; Conlan, S.; Cheley, S.; Bayley, H. Nature 1999. $398,686-689$.

(13) Gu, L. Q.; Serra, M. D.; Vincent, J. B.; Vigh, G.; Cheley, S.; Braha, O.; Bayley, H. Proc. Natl. Acad. Sci. U.S.A. 2000, 97, 39593964.

(14) Gu, L. Q.; Cheley, S.; Bayley, H. Science 2001, 291, 636-640.

(15) Storm, A. J.; Chen, J. H.; Ling, X. S.; Zandbergen, H. W.; Dekker, C. Nature Materials 2003, 2, 537-540.
(16) Chang, H.; Kosari, F.; Andreadakis, G.; Vasmatzis, G.; Basgall, E.; King, A. H.; Bashir, R. Proceedings of the 2004 Hilton Head MEMS Conference, Hilton Head, SC, 2004.

(17) Austin, R. H. Nature Materials 2003, 2, 567-568.

(18) Parks, G. A. Chem. Rev. 1965, 65, 177-198.

(19) Raiteri, R.; Margesin, B.; Grattarola, M. Sens. Actuators B 1998, $46,126-132$.

(20) It should be noted that the use of a silicon nitride pore, as described in earlier study, ${ }^{8,9}$ will not have a measurable interface current since the silicon nitride surface is almost neutral around $\mathrm{pH} 7$ (known to have both silanol and amine groups at the surface at roughly equal densities). ${ }^{19,21}$

(21) Harame, D. L.; Bousse, L. J.; Shott, J. D.; Meindle, J. D. IEEE Trans. Electron Dev. 1987, 34, 1700-1706.

(22) These currents and the dimensions of the channel correspond to resistivity of $45-58 \Omega \mathrm{cm}$, and these values are close to the theoretical value of $78 \Omega \mathrm{cm}$ for $0.1 \mathrm{M} \mathrm{KCl}$ solution. In this calculation, we assume the voltage drop across the pore to be 0.67 of the voltage applied at the electrodes.

(23) Daiguji, H.; Yang, P.; Majumdar, A. Nano Lett. 2004, 4, 137-142.

(24) Since the mobility of potassium ions in the accumulated channel is known and can be expected to be $3-4 \times$ lower that the bulk mobility, the calculated accumulation current can range from $\sim 28$ to $84 \mathrm{pA}$. Since the blocking current was calculated to be about 19 pA lower than the baseline, the total current could range from 9 to $65 \mathrm{pA}$. The value of mobility will be independently measured in future experiments.

NL049267C 